\title{
Sigma Meson Cloud And Proton'S Light Flavor Sea Quarks
}

\author{
Feng Huang ${ }^{\mathrm{a}}$, Rong-Guang $\mathrm{Xu}{ }^{\mathrm{a}}$, Bo-Qiang Ma ${ }^{\mathrm{b}, *}$ \\ ${ }^{a}$ Department of Physics, Peking University, Beijing 100871, China \\ ${ }^{\mathrm{b}}$ CCAST (World Laboratory), P.O. Box 8730, Beijing 100080, China \\ Department of Physics, Peking University, Beijing 100871, China
}

\begin{abstract}
We take into account the sigma meson cloud effect in the meson cloud model to calculate the distributions of light flavor sea quarks in the proton. Our calculation gives a better description of the data for $\bar{d}(x) / \bar{u}(x)$. We also provide a picture that the probability of finding a physical proton in a Fock state $|N \omega\rangle$ is reasonable small with a smaller cutoff $\Lambda_{\omega}$.
\end{abstract}

Key words: Sigma meson, Light flavor sea quarks, Meson cloud model, Meson exchange model

PACS: 11.30.Hv, 12.39.-x, 13.60.-r, 14.40.-n

By now, it has been experimentally established that the light-flavor sea quarks $\bar{d}(x)$ and $\bar{u}(x)$ in the proton differ substantially $[1,2,3,4]$. The violation of the Gottfried sum rule, first found by NMC [1], indicated that $D \equiv \int_{0}^{1}[\bar{d}(x)-$ $\bar{u}(x)] \mathrm{d} x=0.148 \pm 0.039$, and similar result for $\bar{d}(x)-\bar{u}(x)$ was obtained by HERMES [2]. In Drell-Yan experiments, $\bar{d} / \bar{u}$ was determined to be more than 2 at $x=0.18$ by NA51 [3] and the Bjorken- $x$ dependence (moment fraction dependence) of the ratio has been measured by E866/NuSea [4].

While no known symmetry requires $\bar{d}(x)$ equal to $\bar{u}(x)$, the large asymmetry was out of naive expectations. The sea of light flavor quark-antiquark pairs produced perturbatively from gluon splitting is flavor symmetric. Thus the large asymmetry requires a nonperturbative origin. It was first predicted by Thomas [5], and later suggested by Henley and Miller [6], and Kumano

* Corresponding author.

Email address: mabq@phy.pku.edu.cn (Bo-Qiang Ma). 
and Londergan [7], that including the effects of virtual mesons (recognized as meson cloud model) can naturally account for the asymmetry. In previous articles applying meson cloud model to explain the light flavor sea asymmetry in the nucleon, a good description of the data was obtained for the distribution functions [8]. The dominant role is played by the pion, which provides that $\bar{d}(x) / \bar{u}(x)$ either increases monotonically with $x$ or turns back towards unity too slowly $[8,9]$. Many explanations have been applied to attack this problem $[10,11,12,13]$, such as effects of $\Delta[10,11,12]$, the influence of the Pauli exclusion principle [12], adjustment of parameters [13], but none of these explanations provides a satisfactory description of the ratio $\bar{d}(x) / \bar{u}(x)$. However, Alberg, Henley and Miller [14] found that the inclusion of the effect of the isoscalar vector meson $\omega$, with a coupling constant $g_{\omega}^{2} / 4 \pi \approx 8.1$, allows a good description of the data. As mentioned in that article, adding the $\sigma$ effect will likely improve the description of the data. Here, we follow this suggestion and take into account the sigma meson effect in the meson cloud model.

The existence of the $\sigma$ meson has been obscure for many years, while many theorists and experimentalists were searching for $\sigma$ which may play an important role in nuclear physics, because the $\sigma$ meson can provide reasonable middle-range nuclear force [15]. Sigma meson was first introduced as the chiral partner of pion in the linear representation of chiral symmetry, which is an important ingredient in modern hadron physics. It also plays an important role in spontaneous chiral symmetry breaking to understand the present spectroscopy of hadrons. However, it was not well established experimentally mainly due to the negative results of extensive analysis of the $\pi$ - $\pi$ scattering phase shift. While recent reanalysis of the $\pi-\pi$ scattering phase shift [16] strongly suggested a pole of the $\sigma$-meson. Though we do not have a confirmed conclusion that the sigma meson exists as a real physical particle, it is sufficient for considering its effect to calculate $\bar{d}(x)-\bar{u}(x)$ and $\bar{d}(x) / \bar{u}(x)$ in the proton in the meson cloud model.

In the meson cloud model $[7,8,9]$, the nucleon can be viewed as a bare nucleon (core) plus a series of baryon-meson Fock states which result from the fluctuation of nucleon to baryon plus meson $N \rightarrow B M$ (a bare baryon surrounded by a meson cloud). The model assumes that the life-time of a virtual baryon-meson Fock state is much longer than the interaction time in deep inelastic scattering (DIS) or Drell-Yan processes, thus the quark and anti-quark in the virtual baryon-meson Fock states can contribute to the parton distribution of the nucleon. These non-perturbation contribution can be calculated in a convolution between the fluctuation function, which describes the microscopic process $N \rightarrow B M$, and the quark (anti-quark) distribution of hadrons in the Fock states $|B M\rangle$. Here we provide the usual formula. First the physical proton wave function is composed of the following Fock states 


$$
|P\rangle=\sqrt{Z}|P\rangle_{\text {bare }}+\sum_{B M} \int \mathrm{d} y \mathrm{~d}^{2} \mathbf{k}_{\perp} \Psi_{B M}\left(y, \mathbf{k}_{\perp}^{2}\right)\left|B\left(y, \boldsymbol{k}_{\perp}\right), M\left(1-y,-\boldsymbol{k}_{\perp}\right)\right\rangle .
$$

Here $Z$ is the wave function renormalization constant, $\Psi_{B M}\left(y, \mathbf{k}_{\perp}^{2}\right)$ is the probability amplitude for finding a physical nucleon in a state consisting of a baryon $B$ with longitudinal moment fraction $y$ and transverse momentum $\boldsymbol{k}_{\perp}$, and a meson $M$ with longitudinal moment fraction $1-y$ and transverse momentum $-\boldsymbol{k}_{\perp}$. Therefore, the quark distribution functions $q(x)$ in the proton are given by

$$
q(x)=Z q_{\text {bare }}(x)+\delta q(x)
$$

with

$$
\delta q(x)=\sum_{M B}\left[\int_{x}^{1} \frac{\mathrm{d} y}{y} f_{M B}(y) q_{M}\left(\frac{x}{y}\right)+\int_{x}^{1} \frac{\mathrm{d} y}{y} f_{B M}(y) q_{B}\left(\frac{x}{y}\right)\right],
$$

and

$$
f_{M B}(y)=f_{B M}(1-y)
$$

As shown in [11], the wave function renormalization constant reads

$$
Z=\left[1+\sum_{B M} \int_{0}^{1} f_{B M}(y) \mathrm{d} y\right]^{-1} \simeq 1-\sum_{B M} \int_{0}^{1} f_{B M}(y) \mathrm{d} y .
$$

The number of each type of meson, $n_{M}$, is obtained by integrating $f_{M B}(y)$ over $y$. Then we have $Z=1-\sum_{M} n_{M}$. The splitting function is

$$
f_{B M}(y)=\int_{0}^{\infty}\left|\Psi_{B M}\left(y, \mathbf{k}_{\perp}^{2}\right)\right|^{2} \mathrm{~d}^{2} \mathbf{k}_{\perp}
$$

We use time-ordered perturbation theory (TOPT) in the infinite momentum frame (IMF) to calculate this function, which was given by [8] as

$$
f_{B M}(y)=\frac{1}{4 \pi^{2}} \frac{m_{N} m_{B}}{y(1-y)} \frac{\left|G_{M}\left(y, \mathbf{k}_{\perp}^{2}\right)\right|^{2}\left|V_{\mathrm{IMF}}\right|^{2}}{\left[m_{N}^{2}-M_{B M}^{2}\left(y, \mathbf{k}_{\perp}^{2}\right)\right]^{2}},
$$

where 


$$
M_{B M}^{2}\left(y, \mathbf{k}_{\perp}^{2}\right)=\frac{m_{B}^{2}+\mathbf{k}_{\perp}^{2}}{y}+\frac{m_{M}^{2}+\mathbf{k}_{\perp}^{2}}{1-y}
$$

is the invariant mass of the final state, and an exponential form for the cutoff is $[11,15]$

$$
G_{M}\left(y, \mathbf{k}_{\perp}^{2}\right)=\exp \left(\frac{m_{N}^{2}-M_{B M}^{2}\left(y, \mathbf{k}_{\perp}^{2}\right)}{2 \Lambda_{M}^{2}}\right)
$$

which is required to respect Eq.(3). Here $\Lambda_{M}$ is a cutoff parameter for each meson.

The vertex function $V_{\mathrm{IMF}}\left(y, \mathbf{k}_{\perp}^{2}\right)$ depends on the effective interaction Lagrangian that describes the fluctuation process $N \rightarrow B M$. Here we use the meson exchange model for hadron production [15] and the linear sigma model [17] to perform the calculation.

In a full calculation, we should include all kinds of mesons and baryons. While the probability of baryon-meson fluctuation should decrease with the invariant mass of the baryon-meson Fock state increasing, we can neglect the effects of Fock states with higher invariant mass. We just include specifically $\pi, \sigma$ and $\omega$ mesons with the nucleon here. It has been discussed that the effect of the $\rho$ meson and the intermediate $\Delta$ can also contribute a lot each. But the $\rho$ meson increases $\bar{d}(x)-\bar{u}(x)$, whereas the intermediate $\Delta$ decreases it, so these effects tend to cancel each other. And we also omit the effect of $\eta$ for its small coupling constant. Thus it is safe to focus on the effects of $\pi, \sigma$ and $\omega$ meson clouds here.

First, we focus on the sigma meson. From the Bonn meson-exchange model for the nucleon-nucleon interaction [15], the effective interaction Lagrangian for the scalar meson $\sigma$ is $L=g \bar{\psi} \sigma \psi$. Thus we can get the vertex function for $\sigma$ to obtain the fluctuation function as

$$
f_{N \sigma}(y)=\frac{g_{\sigma}^{2}}{8 \pi^{2}} \frac{1}{y^{2}(1-y)} \int_{0}^{\infty} \mathrm{d} \mathbf{k}_{\perp}^{2}\left|G_{\sigma}\left(y, \mathbf{k}_{\perp}^{2}\right)\right|^{2} \frac{m_{N}^{2}(1-y)^{2}+\mathbf{k}_{\perp}^{2}}{\left[m_{N}^{2}-M_{N \sigma}^{2}\left(y, \mathbf{k}_{\perp}^{2}\right)\right]^{2}} .
$$

Now, we should know the coupling constant $g_{\sigma}$, the mass $m_{\sigma}$ and the cutoff $\Lambda_{\sigma}$.

As mentioned above, sigma meson is introduced to the hadron physics as the chiral partner from the view of chiral symmetry. In the $S U(2)$ linear $\sigma$ model [17], the effective Lagrangian is 


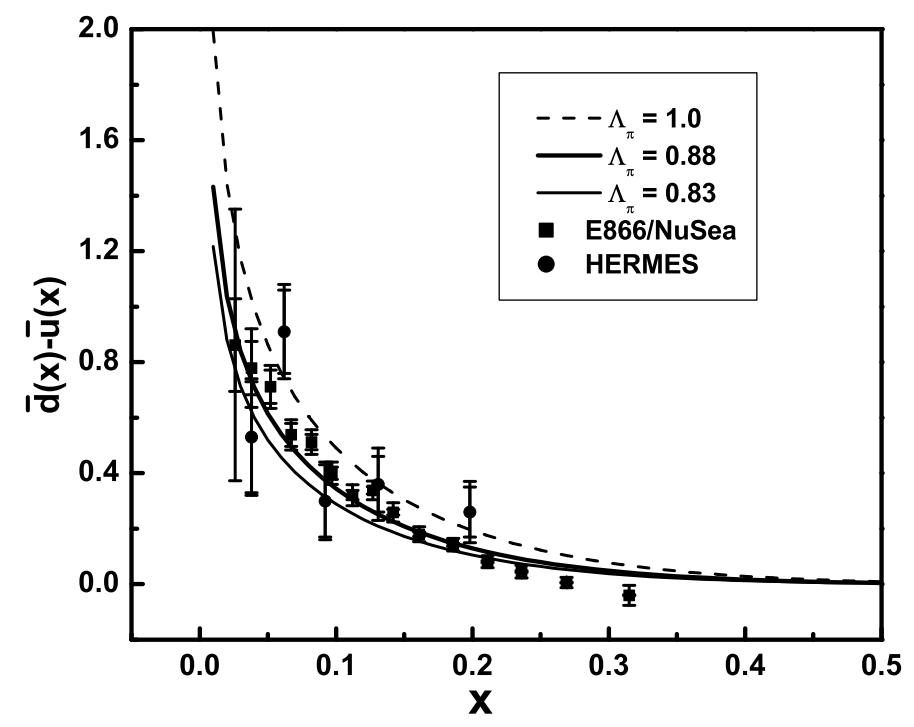

Fig. 1. Comparison of our meson cloud model with data $[2,4]$ for $\bar{d}(x)-\bar{u}(x)$. The thick solid curve is for $\Lambda_{\pi}=0.88 \mathrm{GeV}$, which gives a best description here.

$$
L=g \bar{\psi}\left(\sigma+\gamma_{5} \tau \cdot \pi\right) \psi
$$

Even though we do not have direct information about $g_{\sigma}, g_{\sigma}=g_{\pi}$ is imposed by the linear $\sigma$ model. According to all kinds of calculations to fit different experimental data $[15,18,19]$, the coupling constant $g_{\sigma}^{2} / 4 \pi=g_{\pi}^{2} / 4 \pi=13.6$ is taken in our numerical computations. In 2002 Particle Data Group (PDG), $f_{0}(600)$ or $\sigma$ appears below $1 \mathrm{GeV}$ mass region. Recently it was found that $m_{\sigma}=585 \pm 20 \mathrm{MeV}$ by a reanalysis of $\pi \pi$ scattering phase shift [16]. Similarly, by analysing $\pi \pi$ production processes [16], we obtain $m_{\sigma}=580 \pm 30 \mathrm{MeV}$ for $p p$ central collision. Thus, we will set the mass as $600 \mathrm{MeV}$ in the following calculations. It is generally believed that the cutoff $\Lambda_{\sigma}$ value is in a range around $1 \mathrm{GeV}$ as a phenomenological parameter. Here we will examine the effect of varying $\Lambda_{\sigma}$ value in the range $1.0<\Lambda_{\sigma}<1.3 \mathrm{GeV}$.

We have made some detailed discussions about the $\sigma$ meson cloud above. The $\pi$ meson cloud effect has been discussed enough in previous articles $[6,8,14,20]$, here we just set the parameters as $m_{\pi}=139 \mathrm{MeV}$ and $\Lambda_{\pi}=$ $(0.88 \pm 0.05) \mathrm{GeV}$, which are chosen to reproduce the integrated asymmetry $D \equiv \int_{0}^{1}[\bar{d}(x)-\bar{u}(x)] d x[1,2]$ as shown in Fig. 1 .

Moreover, we should discuss the functions of $q_{M}(x)$ and $q_{B}(x)$. Those for the nucleon and pion are measured, but the quark distribution functions of the $\sigma$ meson and $\omega$ meson are unknown. It has been traditional [21] to assume that the structure function of the $\rho$ and $\pi$ are the same. It is suggested by the 


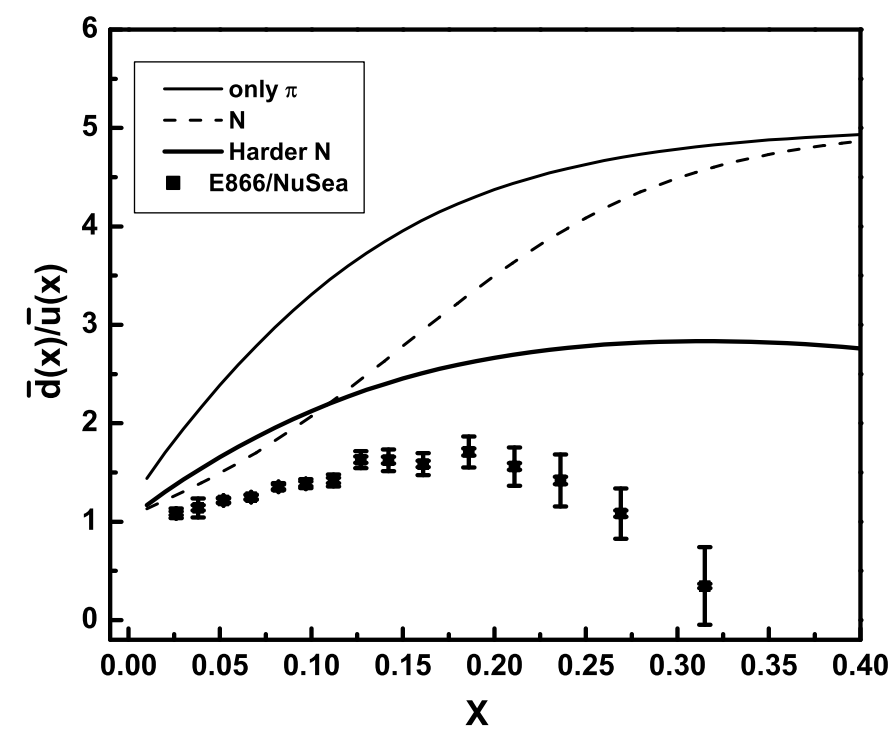

Fig. 2. Comparison of a harder bare nucleon sea quarks (thick solid line) with a traditional bare nucleon sea quarks (dashed line). The thin solid line is only the $\pi$ contribution to the ratio $\bar{d}(x) / \bar{u}(x)$.

bag model [14] that the structure functions of the $\omega, \rho$, and $\pi$ are the same. Due to that the $\sigma$ meson operator is adopted as $\frac{1}{\sqrt{2}}(\bar{u} u+\bar{d} d)$, there is some reason to assume that the quark distribution functions of the $\sigma$ meson is equal to that of the $\pi$. Thus we use [22]

$$
\begin{aligned}
x q_{\mathrm{v}}(x) & =0.99 x^{0.61}(1-x)^{1.02}, \\
x q_{\text {sea }}(x) & =0.2(1-x)^{5.0}
\end{aligned}
$$

for the valence and sea quark distribution functions of all mesons considered here.

The bare nucleon sea is traditionally parametrized as [23]

$$
\begin{aligned}
x \bar{q}_{\text {bare }}(x) & =0.11(1-x)^{15.8}, \\
\bar{q}_{\text {bare }}=u_{\text {sea }} & =\bar{u}_{\text {sea }}=d_{\text {sea }}=\bar{d}_{\text {sea }},
\end{aligned}
$$

which is recognized as Holtmann's parametrization of the bare nucleon symmetric sea. While Alberg and Henley [20] used a harder distribution for the bare sea quarks of the form found in the determination of the gluon distribution [24]

$$
x \bar{q}_{\text {bare }}(x)=0.0124 x^{-0.36}(1-x)^{3.8} .
$$




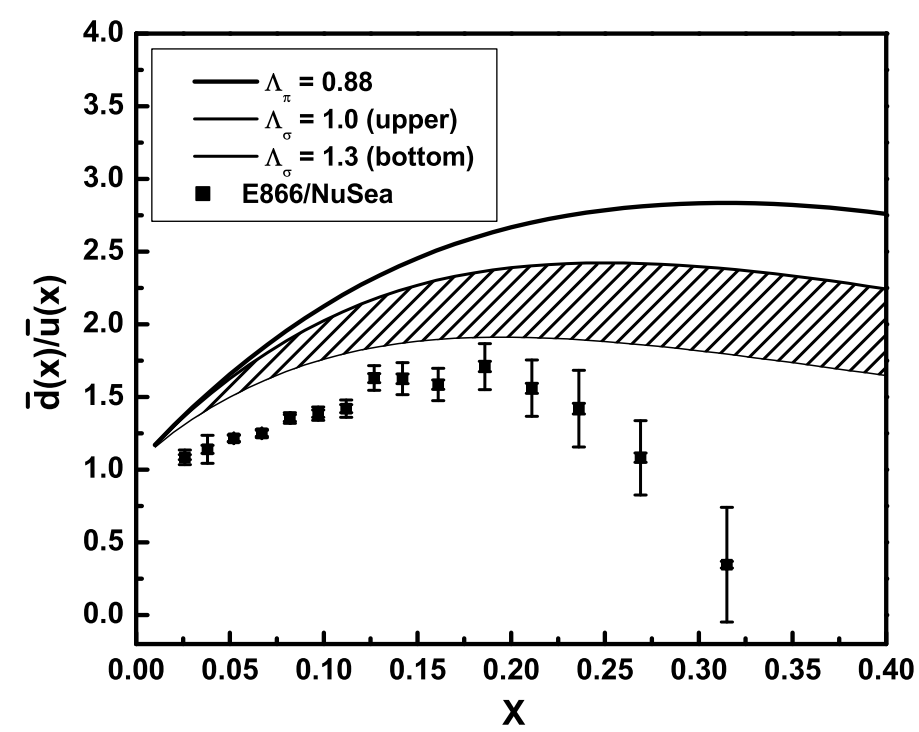

Fig. 3. Comparison of our meson cloud model with data [4] for $\bar{d}(x) / \bar{u}(x)$. The thick solid curve shows our result only considering the $\pi$ meson contribution. The shadowed area shows the effect of adding $\sigma$ meson and varying $\Lambda_{\sigma}$ value in the range $1.0<\Lambda_{\sigma}<1.3 \mathrm{GeV}$.

Their calculations indicate that the harder distribution for the bare sea quarks gives a better description of $\bar{d}(x) / \bar{u}(x)$ as shown in Fig. 2. In the following calculations, we will use only the harder bare nucleon sea. Also we can see that the change is not enough to explain the data, which guarantees the necessary to consider other flavor symmetric contribution. The inclusion of isoscalar meson $\omega$ with a reasonable coupling constant produces a similar improvement in agreement between theory and experiment [14], so does the inclusion of isoscalar meson $\sigma$.

The results of our calculations for light flavor sea quarks are shown in Fig. 3 and Fig. 4. In Fig. 3, we examine the effect of varying $\Lambda_{\sigma}$ value in the range $1.0<\Lambda_{\sigma}<1.3 \mathrm{GeV}$. The thin solid curve on the upper of the shadowed area is the result of setting $\Lambda_{\sigma}=1.0 \mathrm{GeV}$, while the bottom curve corresponds to $\Lambda_{\sigma}=1.3 \mathrm{GeV}$. It is clear that adding sigma meson effect here gives really better description of the data. Our calculations illustrate that the larger value of $\Lambda_{\sigma}$ tends to give small values of the ratio $\bar{d} / \bar{u}$ and that decreasing $\Lambda_{\sigma}$ causes the maximum value of $\bar{d} / \bar{u}$ to be larger and to appear at a higher value of $x$.

In Fig. 4, we illustrate the effects from three mesons with different cutoff values. As calculated in [14], we set $g_{\omega}^{2} / 4 \pi$ as 8.1 , which is from fitting to dispersion relation descriptions of forward nucleon-nucleon scattering $[15,19,25]$. The shadowed area shows the results of varying $\Lambda_{\omega}$ value in the 

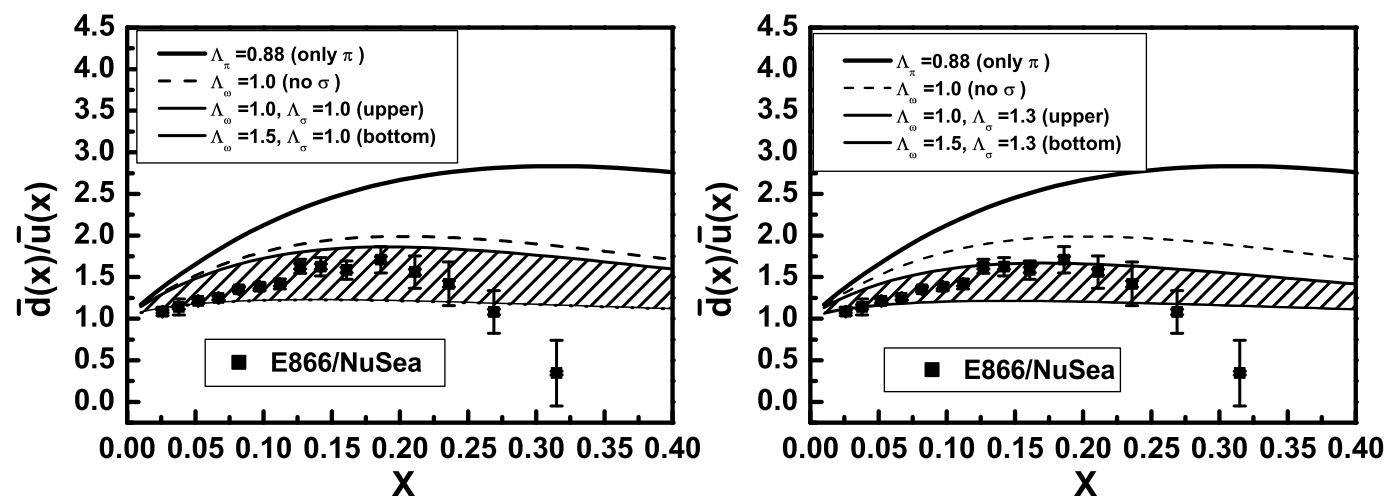

Fig. 4. Comparison of our meson cloud model with data [4] for $\bar{d}(x) / \bar{u}(x)$. The thick solid curve shows our result if contributions from the $\sigma$ and $\omega$ clouds are omitted. The dashed curve shows adding $\omega$ cloud contribution with $\Lambda_{\omega}=1.0 \mathrm{GeV}$. The shadowed area shows the contributions of three mesons, and the effect of varying $\Lambda_{\omega}$ value in the range $1.0<\Lambda_{\omega}<1.5 \mathrm{GeV}$.

range $1.0<\Lambda_{\omega}<1.5 \mathrm{GeV}$ with $\Lambda_{\sigma}$ at $1.0 \mathrm{GeV}$ on the left and $1.3 \mathrm{GeV}$ on the right. The dashed curves omit the $\sigma$ contribution and only consider the value of $\Lambda_{\omega}$ as $1.0 \mathrm{GeV}$. When the value of $\Lambda_{\omega}$ is $1.5 \mathrm{GeV}$, the ratio $\bar{d}(x) / \bar{u}(x)$ hardly changes with adding $\sigma$ meson cloud, which is consistent with [14], in which $\Lambda_{\omega}$ is set as $1.5 \mathrm{GeV}$ to obtain a good description of data by considering only $\pi$ and $\omega$ effects. In order to have a deep understanding of this, we present the numbers of the related mesons $n_{M}$ in the proton with the different cutoff values in the two works, which are shown in Table 1 . The large value of cutoff leads to a large value of meson number in the proton. Apparently, the too large $n_{\omega}$ resulted from the large value of $\Lambda_{\omega}=1.5 \mathrm{GeV}$ should not be the real picture of the proton. For this reason, $\Lambda_{\omega}$ favors the small value of the examined range. Adding $\sigma$ cloud effect modifies the parameters describing the omega-nucleon interaction. And such a revision provides a picture that the probability of finding a physical proton in a Fock state $|N \omega\rangle$ is reasonably small. We also realize that the same rule holds true for the $\sigma$-meson from the comparison of the two different values of $\Lambda_{\sigma}$.

Table 1. Parameters and meson numbers in the proton

\begin{tabular}{|c|c|c|c|c|c|c|}
\hline & $\Lambda_{\pi}$ & $\Lambda_{\sigma}$ & $\Lambda_{\omega}$ & $n_{\pi}$ & $n_{\sigma}$ & $n_{\omega}$ \\
\hline Our work & 0.88 & $1.0 \sim 1.3$ & $1.0 \sim 1.5$ & 0.175 & $0.023 \sim 0.078$ & $0.063 \sim 0.671$ \\
\hline Alberg-Henley's work & 0.83 & no sigma & 1.5 & 0.150 & no sigma & 0.671 \\
\hline
\end{tabular}

In summary, the inclusion of the $\sigma$ meson cloud effect brings a better de- 
scription for $\bar{d}(x) / \bar{u}(x)$ in the proton and also provides a picture of a reasonable small $n_{\omega}$ in the proton. Thus we conclude that the inclusion of both isoscalar meson sigma and omega cloud effects can give an improved description of the experimental data for $\bar{d}(x) / \bar{u}(x)$ in the meson cloud model.

This work is partially supported by National Natural Science Foundation of China under Grant Numbers 10025523 and 90103007.

\section{References}

[1] NMC Collaboration, P. Amaudruz et al., Phys. Rev. Lett. 66 (1991) 2712;

M. Arneodo et al., Phys. Rev. D50 (1994) R1.

[2] K. Ackerstaff et al., Phys. Rev. Lett. 81 (1998) 5519.

[3] A. Baldit et al., Phys. Lett. B332 (1994) 244.

[4] E866 Collaboration, E.A. Hawker et al., Phys. Rev. Lett. 80 (1998) 3715;

J.C. Peng et al., Phys. Rev D58 (1999) 092004;

R.S. Towell et al., Phys. Rev D64 (2001) 052002.

[5] A.W. Thomas, Phys. Lett. B126 (1983) 97.

[6] E.M. Henley, G.A. Miller, Phys. Lett. B251 (1990) 453.

[7] S. Kumano, Phys. Rev. D43 (1991) 3067;

S. Kumano, J.T. Londergan, Phys. Rev. D44 (1991) 717.

[8] For reviews, see, e.g., S. Kumano, Phys. Rep. 303 (1998) 183;

G.T. Garvey, J.C. Peng, Prog. Part. Nucl. Phys. 47 (2001) 203.

[9] M. Alberg, T. Falter, E.M. Henley, Nucl. Phys. A644 (1998) 93.

[10] A. Signal, A.W. Schreiber, A.W. Thomas, Mod. Phys. Lett. A6 (1991) 271.

[11] W. Koepf, L.L. Frankfurt, M. Strikman, Phys. Rev. D53 (1996) 2586.

[12] W. Melnitchouk, J. Speth, A.W. Thomas, Phys. Rev. D59 (1998) 014033.

[13] N.N. Nikolaev, W. Schäfer, A. Szczurek, J. Speth, Phys. Rev. D 60 (1999) 014004.

[14] M. Alberg, E.M. Henley, G.A. Miller, Phys. Lett. B471 (2000) 396.

[15] R. Machleidt, K. Holinde, and Ch. Elster, Phys. Rep. 149 (1987) 1.

[16] M. Ishida, hep-ph/9905261. 
[17] A. Hosaka, H. Toki, Quarks, Baryons and Chiral Symmetry, World Scientific, (2001).

[18] R.J. Fries, A. Schäfer, C. Weiss, Eur. Phys. J. A17 (2003) 509.

[19] Y.-B. Ding et al., J. Phys. G 30 (2004) 841.

[20] M. Alberg, E.M. Henley, Nucl. Phys. A663 (2000) 301.

[21] W.Y.-P. Hwang, J. Speth, G.E. Brown, Z. Phys. A339 (1991) 383;

A. Szczurek, J. Speth, Nucl. Phys. A555 (1993) 249.

[22] P.J. Sutton, A.D. Martin, R.G. Roberts, W.J. Stirling, Phys. Rev. D45 (1992) 2349 .

[23] H. Holtmann, Mesonen im Nukleon und ihre Auswirkungen in elastischer und tiefinelastischer Streung, Forschungszentrum Juelich, Dissertation, University of Bonn (1995).

[24] A. Vogt, hep-ph/9807369.

[25] P.C. Tandy, hep-ph/9808029. 